\title{
Reading Between the Lines: Exploring Lexical and Emotional Content in Baseline Trauma Impact Statements
}

\author{
Jeffrey M. Girard ${ }^{1}$, Joel G. Sprunger ${ }^{2}$, and Kathleen M. Chard ${ }^{2,3}$ \\ ${ }^{1}$ Department of Psychology, University of Kansas \\ ${ }^{2}$ Department of Psychiatry and Behavioral Neuroscience, University of Cincinnati \\ ${ }^{3}$ Cincinnati VA Medical Center, Trauma Recovery Center, Cincinnati, Ohio
}

\begin{abstract}
The words a person uses while describing salient life events/experiences can provide a wealth of information about their cognitive and emotional functioning. Linguistic analyses can thus play an important role in clinical settings by providing insights into patients' mental health. The current paper applies such techniques to the study of posttraumatic stress disorder (PTSD). It uses a combination of computational and human rating methods to analyze the lexical and emotional content of trauma appraisal essays (i.e., impact statements) written by a treatment-seeking clinical sample $(\mathrm{N}=94)$. More frequent use of first-person plural pronouns (e.g., 'we', 'our') was associated with lower self-reported PTSD and depressive severity, higher ratings of expressed anger were associated with higher clinician-rated PTSD severity, and higher ratings of expressed vulnerable emotions were associated with higher self-reported PTSD and depressive severity. Text-mining approaches also provided insights into common themes across participants' trauma impact statements (e.g., safety, trust, and self-esteem).
\end{abstract}

Keywords: linguistics; posttraumatic stress disorder; cognition(s); emotion

Posttraumatic Stress Disorder (PTSD) is a serious mental health concern that creates psychological suffering, impairs daily living, and conveys risk for negative outcomes including depression, anger problems, and suicide (McKinney, Hirsch, \& Britton, 2017). Prior to the COVID-19 pandemic, the lifetime prevalence of PTSD among American civilians was estimated at $6 \%$ overall and reached as high as $27 \%$ in specific demographic groups (Schein et al., 2021). However, with over 800,000 lives lost to COVID-19 in the US alone, the general rates of exposure to sudden life-threatening

Open data, open materials, and supplemental materials are available from: osf.io/fxksw. We have no conflicts of interest to report. We thank our participants for their courage and generosity and our research staff for their diligent and careful work. JMG and JGS contributed equally to the study.

Correspondence concerning this article should be addressed to Jeffrey Girard, 1415 Jayhawk Blvd, Lawrence, KS 66045. Email: jmgirard@ku.edu illness and death have inevitably increased. Thus, the already critical imperative to study, detect, and treat PTSD is only growing in importance and relevance.

Cognitive theories of PTSD (e.g., Ehlers \& Clark, 2000) propose that its development and maintenance are greatly influenced by the way one thinks about traumatic events, e.g., the attributions one makes about their causes and consequences/implications. These attributions are thought to be the products of a meaningmaking process that seeks to reduce discrepancies between the trauma's occurrence and one's understanding of oneself, other people, and the world (Park, 2010). If this process results in distorted cognitions, significant distress can be elicited through activation of negative affect and autonomic arousal. Two common types of distorted cognitions in PTSD are assimilation, when appraisals of the event are changed to fit existing beliefs (e.g., "if bad things don't happen to good people, I must not be a good person"), and overaccommodation, when the lessons learned from the event are overgeneralized (e.g., "everyone will take advantage of me"). Further, 
distorted cognitions can contribute to the maintenance of PTSD by encouraging the systematic avoidance of trauma-related thoughts, feelings, and cues, as avoidance can block natural recovery mechanisms such as corrective re-appraisals, exposure experiences, and use of social support (Ehlers \& Clark, 2000).

Writing samples are a rich source of information about patients' trauma-related cognitions and emotions. Previous research found that the words people use while discussing adverse experiences can reveal their current level of psychological distress and predict later mental health outcomes (Pennebaker, Mehl, \& Niederhoffer, 2003). In the domain of PTSD, past work focused on analyzing patients' trauma narratives using closedvocabulary approaches (Eichstaedt et al., 2021), which count the proportion of words in a text that fall into predefined lexical categories. Commonly explored categories in the PTSD literature include positive emotion words, negative emotion words, words related to cognitive processing, words related to death, and first-person pronouns. The pattern of results across these studies has been somewhat equivocal (e.g., Jaeger, Lindblom, Parker-Guilbert, \& Zoellner, 2014; Miragoli, Camisasca, \& Di Blasio, 2019; Papini, Yoon, Rubin, Lopez-Castro, \& Hien, 2015), but the fact that many of these measures have predicted current PTSD symptom severity and longitudinal outcomes within individual studies suggests that this method has potential (Kleim, Horn, Kraehenmann, Mehl, \& Ehlers, 2018).

Several limitations of the previous work are worth noting. First are limitations of the methods used to sample participants and assess their symptoms. Previous studies primarily recruited from non-clinical settings and focused on specific trauma types such as physical and sexual assault (e.g., Jaeger et al., 2014; Miragoli et al., 2019). As a result, it is unclear how well their findings will generalize to more severely impaired, clinical populations and individuals who experienced other trauma types. Many previous studies also relied on selfreport measures of symptoms rather than gold-standard clinical interviews.

Second are limitations of the methods used to elicit language samples from participants. Nearly all previous work examined trauma narratives, which are constrained to discussing negative experiences in the past and therefore may be limited in terms of the affective and cognitive processes they reveal. A promis- ing alternative is to collect trauma appraisals, which are verbal descriptions of how the individual views the antecedents, consequences, and meaning of traumatic events. Several pioneering studies analyzed trauma appraisals, predicting current symptom severity (Sobel, Resick, \& Rabalais, 2009) and longitudinal symptom trajectory (Iverson, King, Cunningham, \& Resick, 2015) from ratings of assimilated and overaccommodated thoughts in the essays. However, no research has examined the affective content of trauma appraisals despite the important relationships between appraisals, emotions, and symptoms.

Third are limitations of the methods used to quantify participants' language usage patterns. Closedvocabulary approaches ignore context by considering each word in isolation (e.g., the word 'sad' in both 'very sad' and 'not sad' would increment the negative emotion category), ignore semantic intensity by treating each word in a category as equal (e.g., both 'annoyed' and 'enraged' would count the same), and are highly influenced by a small number of high-frequency words in each category (Eichstaedt et al., 2021). Additionally, when closed-vocabulary approaches are the only method used to explore language, more qualitative insights are often overlooked.

The current study explores the potential of linguistic analysis to facilitate PTSD assessment. We extend the literature and address the limitations of previous work by: (1) recruiting a treatment-seeking clinical sample with many different trauma types, (2) assessing symptoms using both self-report checklists and gold-standard structured interviews, (3) eliciting language samples using trauma appraisals, (4) comparing closed- with open-vocabulary approaches and manual ratings of emotional content, and (5) analyzing the data with robust, Bayesian statistical techniques. We test for correlations between symptoms and word categories supported in previous research (i.e., positive emotion words, negative emotion words, insight words, death words, and first-person pronouns) and hypothesize that higher ratings of expressed negative emotion will be associated with worse symptoms. We also explore several other word categories that we hypothesize will reflect symptoms/cognitive distortions in trauma appraisals (i.e., total word count, past focus words, future focus words, and certainty words). 


\section{Methods}

\section{Participants}

Participants were sampled from an archival clinic dataset of adults with a DSM-5 diagnosis of PTSD that had received Cognitive Processing Therapy (CPT; Resick, Monson, \& Chard, 2017) in an American community-based trauma clinic. Participants were included if their medical file contained their baseline impact statement essay. The final sample consisted of 94 participants (74 female, 20 male). Participant ages ranged from 19 to $68(M=39.1, S D=13.0)$, most identified as White (77 White, 15 Black, 2 Other), most did not have a military service history ( 81 no, 10 yes), and most had some college education (6 some high school, 27 high school degree, 3 vocational school, 33 some college, 16 bachelors, 6 masters). Index traumas included childhood sexual abuse $(n=16)$, transportation accident ( $n=13)$, sexual assault $(n=12)$, physical assault $(n=8)$, witness to death $(n=8)$, childhood physical abuse $(n=8)$, domestic violence $(n=7)$, life-threatening illness or injury $(n=6)$, sudden unexpected death $(n=5)$, assault with a weapon $(n=4)$, fire or explosion $(n=2)$, serious accident $(n=2)$, and witness to suicide attempt $(n=2)$.

\section{Procedure}

Participants completed clinical measures of symptoms related to depression and PTSD at their intake appointment as part of standard clinical practice. As homework after the first session of $\mathrm{CPT}^{1}$, patients handwrote a baseline impact statement essay: a one-page description of what they believe was the cause of their traumatic experience(s) and how these experiences impacted their beliefs about themselves, others, and the world (esp. in the domains of safety, trust, power, control, esteem, and intimacy). We digitized the handwritten impact statements by having one of three transcribers manually type each essay into an electronic text file. Inter-transcriber reliability was assessed by having 15 essays independently transcribed by two different transcribers (i.e., five essays for each pair). Text similarity was calculated by comparing the observed Levenshtein distance to the maximum possible Levenshtein distance (van der Loo, 2014); similarity scores can range from 0.0 (complete dissimilarity) and 1.0 (complete similarity). Reliability was nearly perfect with a mean similarity of $0.98,95 \%$ CI: [0.96, 0.99].

\section{Clinical Measures}

The Clinician-Administered PTSD Scale for DSM5 (CAPS-5) was used to measure clinician-rated PTSD symptom severity (Weathers, Blake, et al., 2013). For each of 30 items, the clinician assigns a past-month severity score to a trauma-related symptom. The first 20 items are summed to calculate the total score.

The PTSD Checklist for DSM-5 (PCL-5) was used to measure patient-reported PTSD symptom severity (Weathers, Litz, et al., 2013). For each of 20 items, the patient indicates the degree to which a trauma-related symptom has bothered them in the past month. All items are summed to calculate the total score.

The Patient Health Questionnaire-9 (PHQ-9) was used to measure patient-reported depressive symptom severity (Kroenke, Spitzer, \& Williams, 2001). For each of nine items, the patient indicates how often a depressive symptom caused distress in the past two weeks. All items are summed to calculate the total score.

\section{Text Mining}

To explore the essays' thematic content, we used text mining (Silge \& Robinson, 2021). To prepare the transcripts for text mining, we used the following preprocessing steps. We first replaced all contractions (e.g., 'didn't') with their constituent words (e.g., 'did not'). We then lemmatized all words by replacing words' inflected forms (e.g., 'walked' and 'walking') with their base forms (e.g., 'walk'). Next, we tokenized the essays into $n$-grams, i.e., sequences of $n$ consecutive words. For example, the string 'the quick brown fox' could be tokenized into unigrams (e.g., 'the' and 'quick'), bigrams (e.g., 'the quick' and 'quick brown'), and trigrams (i.e., 'the quick brown' and 'quick brown fox'). Finally, we removed all $n$-grams that included stop words, i.e., common words that do not convey thematic information (e.g., 'a', 'the', 'and').

\section{Language Measures}

Lexical Measures. To quantify the essays' lexical content, we used the Linguistic Inquiry and Word Count (LIWC) software, which scans through each word in a

\footnotetext{
${ }^{1}$ The first session is focused on psychoeducation, and formal intervention does not begin until the second session.
} 
text and counts the occurrence of words in 90 predefined categories (Pennebaker, Boyd, Jordan, \& Blackburn, 2015). We selected six primary categories based on previous literature: positive emotion words (e.g., 'love', 'nice', 'sweet'), negative emotion words (e.g., 'hurt', 'ugly', 'nasty'), insight words (e.g., 'think', 'know'), death words (e.g., 'bury', 'coffin', 'kill'), singular first-person pronouns (e.g., 'I', 'me', 'mine'), and plural first-person pronouns (e.g., 'we', 'us', 'our'). We also explored four categories without prior literature support: word count (the total number of words), past focus words (e.g., 'ago', 'did', 'talked'), future focus words (e.g., 'may', 'will', 'soon'), and certainty words (e.g., 'always', 'never', 'definitely').

Emotion Ratings. To quantify the essays' emotional content, between four and six $(M=4.5, S D=0.8)$ human raters independently read and rated each essay on the intensity of several emotions using formr (Arslan, Walther, \& Tata, 2020). Ratings were made by answering eight questions, each of which had the stem "How much does this person seem to be feeling..." and was rated on an ordinal scale from 0 (not at all) to 4 (extremely). We selected the following emotions to rate based on literature review and pilot testing: negative emotion, anger, fear/anxiety, hopeless/pessimistic, lonely/alone, sadness/loss, shame/guilt, and powerless/ incompetent. To avoid rater fatigue, raters completed this task in 10 separate batches. The assignment of essays to batches was randomized once for all raters, and the ordering of essays within each batch was randomized for each rater separately. Raters were blind to the essays' authors.

To assess the inter-rater reliability of the emotion ratings, we used the average-measure agreement intraclass correlation coefficient (McGraw \& Wong, 1996). Inter-rater reliability was good ( $>0.75)$ for anger, fearl anxiety, hopeless/pessimistic, powerless/incompetent, and shame/guilt but only moderate $(>0.50)$ for negative emotion, lonely/alone, and sadness/loss (see the supplemental materials). We removed the less-reliable emotions from analysis (in supplemental sensitivity analyses, including them did not alter the results).

\section{Data Analysis}

To test our hypotheses, two complementary approaches were used. Both were completed in the Bayesian statistical framework (Gelman et al., 2014).
We used posterior medians as our point estimates and 95\% highest density intervals (HDIs) as our interval estimates. For each parameter of interest, we also calculated the probability of direction $(p d)$, which ranges from $50 \%$ to $100 \%$ and can be interpreted as the probability that a parameter is strictly positive or negative. We interpreted effects with $p d$ values at or above $95 \%$ as "statistically significant."

First, we examined the bivariate correlations between variables of interest (i.e., clinical measures, LIWC category scores, and emotion ratings) in order to quantify their zero-order relationships. Gaussian rank correlations $(\hat{\rho})$, which are more robust to non-normal distributions and outliers than Pearson correlations (Boudt, Cornelissen, \& Croux, 2012), were estimated using regularizing priors to manage familywise error. These correlational analysis were completed using the correlation package (Makowski, Ben-Shachar, Patil, \& Lüdecke, 2020).

Second, we used structural equation modeling (SEM) to estimate latent variables underlying the emotion rating scales ${ }^{2}$ and regress the clinical measure on those latent variables (Merkle \& Rosseel, 2018). Weakly informative default priors were used for all model parameters and are described in supplemental materials. Model fit was evaluated using the BRMSEA, BGammaHat, and BMc indices (Garnier-Villarreal \& Jorgensen, 2020). We adopted the following heuristics: values of BRMSEA <.08 are 'acceptable' and values $<.05$ are 'good,' and values of BGammaHat and BMc $>.90$ are 'acceptable' and values $>.95$ are 'good.'

\section{Results}

\section{Clinical Measures}

CAPS-5 total scores ranged from 15 to 65 ( $M=44.6$, $S D=10.7)$. PCL-5 total scores ranged from 14 to 80 ( $M=56.0, S D=13.9,2$ missing). PHQ-9 total scores ranged from 2 to 27 ( $M=18.1, S D=6.0,2$ missing). All three measures were significantly and positively intercorrelated (all $p d=100 \%$ ): the CAPS-5 and PCL-5 total scores were correlated at 0.57 (95\% HDI: [0.42, 0.70]), the CAPS-5 and PHQ-9 total scores were correlated at $0.46[0.29,0.60]$, and the PCL-5 and PHQ-9 total scores were correlated at $0.74[0.64,0.83]$.

\footnotetext{
${ }^{2}$ We did not use SEM for the LIWC variables because none of their inter-correlations were strong.
} 


\section{Text Mining}

The essays ranged in length from 31 to 1447 words $(M=367.9, S D=277.3)$. Figure 1a shows the 50 most common unigrams and Figure $1 \mathrm{~b}$ shows the 30 most common bigrams in a directed graph; the graph can be read from node-to-node in the direction of the arrows (e.g., 'too' $\rightarrow$ 'much' can be read as 'too much'). The four most common trigrams were 'not feel safe,' 'not trust anyone,' 'hard time trust,' and 'low self esteem.'

\section{Lexical Measures}

Table 1 reports the correlations between the clinical measures and the LIWC category scores. The CAPS-5 total score was not significantly correlated with any LIWC category scores. The PCL-5 total score was significantly and negatively correlated with plural firstperson pronouns but was not significantly correlated with the other LIWC scores. The PHQ-9 total score was also significantly and negatively correlated with plural first-person pronouns but was not significantly correlated with the other LIWC scores. Thus, participants who self-reported worse PTSD or depression symptoms tended to use fewer words like 'we', 'us', and 'our' .

\section{Emotion Ratings}

According to the third-party raters, the emotions expressed most strongly in the essays were powerless/ incompetent $(M=2.6, S D=0.7)$, fear/anxiety $(M=2.5$, $S D=0.8)$, and hopeless/pessimistic $(M=2.5, S D=0.8)$. The emotions of shame/guilt $(M=2.1, S D=1.0)$ and anger $(M=1.7, S D=0.9)$ were expressed to a lesser degree. The top section of Table 2 reports the correlations among the emotion rating variables, which organized into two clusters. First, the fear/anxiety, hopeless/ pessimistic, shame/guilt, and powerless/incompetent variables were positively inter-correlated. Second, the anger variable stood apart and was only weakly correlated with the other emotion variables. The bottom three sections of Table 2 report the correlations between the clinical measures and the emotion rating variables. The CAPS-5 total score was significantly and positively correlated with the anger rating variable and the shame/ guilt rating variable. The PCL-5 total score was significantly and positively correlated with the hopeless/ pessimistic rating variable. Finally, the PHQ-9 total score was positively and significantly correlated with the hopeless/pessimistic rating variable, the powerless/ incompetent rating variable, and the fear/anxiety rating variable. All other relationships were not significant.

\section{Structural Equation Modeling}

Based on the pattern of correlations among the emotion rating variables, we built an SEM model with two latent variables: one that included the anger rating variable as a single-indicator (i.e., $F_{1}$ ) and another that included the fear/anxiety, hopeless/pessimistic, shame/ guilt, and powerless/incompetent rating variables as indicators (i.e., $F_{2}$ ). All observed and latent variables were standardized to have zero mean and unit variance. To estimate the single-indicator $F_{1}$, we fixed its loading to 1.0 and its error variance to 0.19 (based on Brown, 2015, pp. 122-125). We then regressed the observed clinical measures on both latent variables.

We report the full results of the SEM model in the supplemental materials and summarize the most important parts here and in Figure 2. This model had good fit for two of our indices (BGammaHat $=0.979$ and $\mathrm{BMc}=0.958)$ and acceptable fit for the other (BRM$\mathrm{SEA}=0.079)^{3}$. All loadings on $F_{2}$ were positive and significant $(p d=100 \%)$; they were particularly strong for the powerless/incompetent and hopeless/pessimistic rating variables. The two latent variables were nearly orthogonal/uncorrelated $(r=0.04, p d=62.0 \%)$. Given the pattern of loadings, we named $F_{1}$ 'Expressed Anger' and $F_{2}$ 'Expressed Vulnerability.'

The regression pathway from $F_{1}$ ('Expressed Anger') to the CAPS-5 total score was significant and positive $(\beta=0.24,95 \%$ HDI: [0.04, 0.46], $p d=98.7 \%)$, but the pathways were not significant to the PCL-5 total score $(\beta=0.09,[-0.12,0.30], p d=79.7 \%)$ or the PHQ-9 total score $(\beta=0.09,[-0.12,0.29], p d=80.3 \%)$. The regression pathways were significant and positive from $F_{2}$ ('Expressed Vulnerability') to the PCL-5 total score $(\beta=0.24,[0.01,0.47], p d=98.0 \%)$ and the PHQ-9 total score $(\beta=0.38,[0.16,0.61], p d=100.0 \%)$, but the pathway to the CAPS-5 total score was not significant $(\beta=0.12,[-0.10,0.34], p d=87.0 \%)$.

\footnotetext{
${ }^{3}$ We also fit a supplemental model with a single factor and five indicators; it had slightly worse fit (BRMSEA $=0.085$, BGammaHat=0.971, BMc=0.942).
} 
(a)

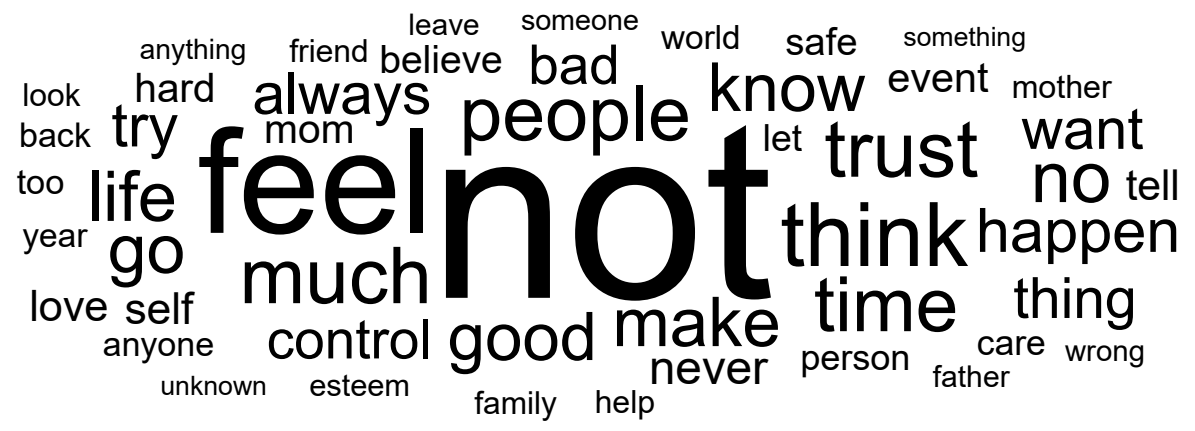

(b)
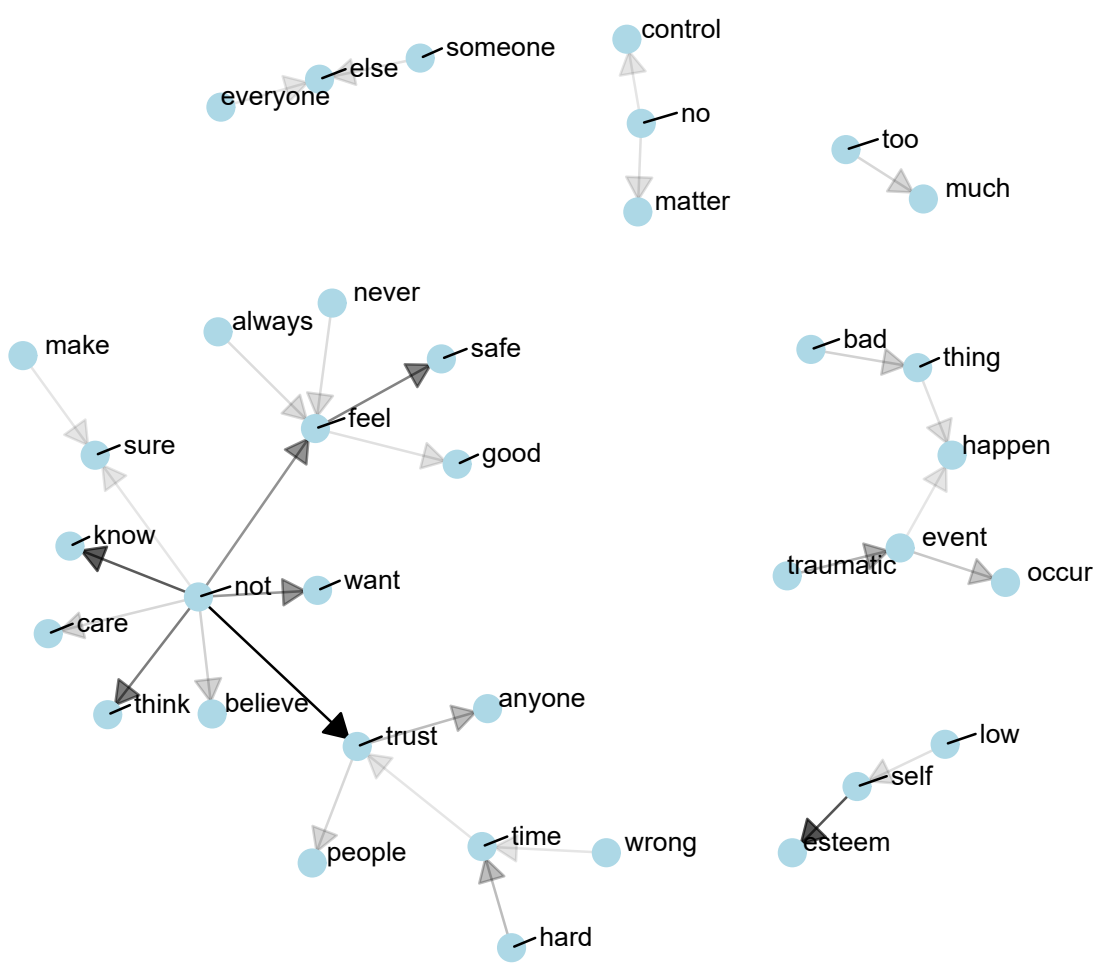

\section{Figure 1}

Visualizations of (a) the 50 most common unigrams with word size indicating relative frequency across all essays and (b) the 30 most common bigrams with arrow opacity/darkness indicating relative frequency. 
Table 1

Correlations Between Clinical Measures and LIWC Category Scores

\begin{tabular}{|c|c|c|c|c|}
\hline Gaussian Rank Correlation & Estimate & $95 \% \mathrm{HDI}$ & $p d$ & Sig. \\
\hline CAPS-5 $\leftrightarrow$ Positive Emotion Words & -0.02 & {$[-0.20,0.19]$} & $58.0 \%$ & \\
\hline CAPS- $5 \leftrightarrow$ Negative Emotion Words & -0.06 & {$[-0.25,0.13]$} & $72.5 \%$ & \\
\hline CAPS-5 $\leftrightarrow$ Insight words & 0.14 & {$[-0.05,0.33]$} & $91.8 \%$ & \\
\hline CAPS-5 $\leftrightarrow$ Death words & -0.15 & {$[-0.35,0.04]$} & $93.3 \%$ & \\
\hline CAPS-5 $\leftrightarrow$ Singular $1^{\text {st }}$ Person Pronouns & 0.13 & {$[-0.05,0.34]$} & $89.6 \%$ & \\
\hline CAPS-5 $\leftrightarrow$ Plural $1^{\text {st }}$ Person Pronouns & -0.05 & {$[-0.24,0.15]$} & $69.0 \%$ & \\
\hline CAPS-5 $\leftrightarrow$ Word Count & 0.08 & {$[-0.11,0.28]$} & $77.7 \%$ & \\
\hline CAPS-5 $\leftrightarrow$ Past Focus Words & 0.11 & {$[-0.09,0.30]$} & $86.6 \%$ & \\
\hline CAPS-5 $\leftrightarrow$ Future Focus Words & -0.08 & {$[-0.27,0.11]$} & $79.8 \%$ & \\
\hline CAPS-5 $\leftrightarrow$ Certainty Words & 0.00 & {$[-0.21,0.18]$} & $51.8 \%$ & \\
\hline PCL-5 $\leftrightarrow$ Positive Emotion Words & 0.07 & {$[-0.11,0.28]$} & $75.9 \%$ & \\
\hline PCL-5 $\leftrightarrow$ Negative Emotion Words & 0.03 & {$[-0.17,0.22]$} & $62.0 \%$ & \\
\hline PCL-5 $\leftrightarrow$ Insight words & 0.08 & {$[-0.11,0.29]$} & $78.1 \%$ & \\
\hline PCL-5 $\leftrightarrow$ Death words & -0.15 & {$[-0.34,0.04]$} & $93.3 \%$ & \\
\hline PCL-5 $\leftrightarrow$ Singular $1^{\text {st }}$ Person Pronouns & 0.08 & {$[-0.12,0.28]$} & $77.9 \%$ & \\
\hline PCL-5 $\leftrightarrow$ Plural $1^{\text {st }}$ Person Pronouns & -0.25 & {$[-0.43,-0.07]$} & $99.6 \%$ & $* *$ \\
\hline PCL-5 $\leftrightarrow$ Word Count & -0.15 & {$[-0.35,0.03]$} & $93.0 \%$ & \\
\hline PCL-5 $\leftrightarrow$ Past Focus Words & 0.11 & {$[-0.09,0.30]$} & $87.8 \%$ & \\
\hline PCL-5 $\leftrightarrow$ Future Focus Words & -0.11 & {$[-0.30,0.10]$} & $85.6 \%$ & \\
\hline PCL-5 $\leftrightarrow$ Certainty Words & 0.12 & {$[-0.08,0.31]$} & $88.0 \%$ & \\
\hline PHQ-9 $\leftrightarrow$ Positive Emotion Words & 0.03 & {$[-0.16,0.23]$} & $61.1 \%$ & \\
\hline PHQ-9 $\leftrightarrow$ Negative Emotion Words & -0.03 & {$[-0.21,0.17]$} & $61.4 \%$ & \\
\hline PHQ-9 $\leftrightarrow$ Insight words & -0.04 & {$[-0.23,0.17]$} & $66.9 \%$ & \\
\hline PHQ-9 $\leftrightarrow$ Death words & -0.07 & {$[-0.27,0.12]$} & $76.9 \%$ & \\
\hline PHQ-9 $\leftrightarrow$ Singular $1^{\text {st }}$ Person Pronouns & 0.06 & {$[-0.14,0.25]$} & $70.9 \%$ & \\
\hline PHQ-9 $\leftrightarrow$ Plural $1^{\text {st }}$ Person Pronouns & -0.25 & {$[-0.42,-0.06]$} & $99.4 \%$ & $* *$ \\
\hline PHQ-9 $\leftrightarrow$ Word Count & -0.10 & {$[-0.31,0.08]$} & $84.1 \%$ & \\
\hline PHQ-9 $\leftrightarrow$ Past Focus Words & 0.11 & {$[-0.10,0.30]$} & $85.9 \%$ & \\
\hline PHQ-9 $\leftrightarrow$ Future Focus Words & -0.05 & {$[-0.25,0.15]$} & $69.3 \%$ & \\
\hline PHQ-9 $\leftrightarrow$ Certainty Words & 0.12 & {$[-0.08,0.31]$} & $87.3 \%$ & \\
\hline
\end{tabular}

Note. ${ }^{*} p d \geq 95 \%,{ }^{* *} p d \geq 99 \%,{ }^{* * *} p d \geq 99.9 \%$

\section{Discussion}

Given the prominent role that cognitions play in the development and maintenance of PTSD, we hypothesized that linguistic analysis of patients' trauma appraisals would yield valuable insights into their psycho- logical functioning at the start of treatment. We used a multi-method approach that combined text mining, closed-vocabulary measures (i.e., LIWC), and human ratings to explore the lexical and emotional content of patients' trauma impact statement essays.

Text mining revealed several key insights into the 
Table 2

Correlations Among Emotion Ratings and Clinical Measures

\begin{tabular}{|c|c|c|c|c|}
\hline Gaussian Rank Correlation & Estimate & $95 \% \mathrm{HDI}$ & $p d$ & Sig. \\
\hline Anger Ratings $\leftrightarrow$ Fear Ratings & -0.01 & {$[-0.19,0.21]$} & $53.2 \%$ & \\
\hline Anger Ratings $\leftrightarrow$ Hopeless Ratings & 0.09 & {$[-0.11,0.28]$} & $81.1 \%$ & \\
\hline Anger Ratings $\leftrightarrow$ Shame Ratings & -0.09 & {$[-0.27,0.11]$} & $81.9 \%$ & \\
\hline Anger Ratings $\leftrightarrow$ Powerless Ratings & -0.06 & {$[-0.25,0.14]$} & $70.5 \%$ & \\
\hline Fear Ratings $\leftrightarrow$ Hopeless Ratings & 0.44 & {$[0.27,0.58]$} & $100.0 \%$ & $* * *$ \\
\hline Fear Ratings $\leftrightarrow$ Shame Ratings & 0.14 & {$[-0.05,0.33]$} & $90.6 \%$ & \\
\hline Fear Ratings $\leftrightarrow$ Powerless Ratings & 0.51 & {$[0.36,0.65]$} & $100.0 \%$ & $* * *$ \\
\hline Hopeless Ratings $\leftrightarrow$ Shame Ratings & 0.35 & {$[0.17,0.52]$} & $100.0 \%$ & $* * *$ \\
\hline Hopeless Ratings $\leftrightarrow$ Powerless Ratings & 0.78 & {$[0.69,0.86]$} & $100.0 \%$ & $* * *$ \\
\hline Shame Ratings $\leftrightarrow$ Powerless Ratings & 0.32 & {$[0.14,0.49]$} & $100.0 \%$ & $* * *$ \\
\hline CAPS-5 $\leftrightarrow$ Anger Ratings & 0.23 & {$[0.05,0.42]$} & $99.3 \%$ & $* *$ \\
\hline CAPS-5 $\leftrightarrow$ Fear Ratings & 0.08 & {$[-0.11,0.28]$} & $80.0 \%$ & \\
\hline CAPS-5 $\leftrightarrow$ Hopeless Ratings & 0.12 & {$[-0.07,0.31]$} & $88.5 \%$ & \\
\hline CAPS-5 $\leftrightarrow$ Shame Ratings & 0.23 & {$[0.03,0.40]$} & $98.9 \%$ & $*$ \\
\hline CAPS-5 $\leftrightarrow$ Powerless Ratings & 0.05 & {$[-0.13,0.26]$} & $70.0 \%$ & \\
\hline PCL-5 $\leftrightarrow$ Anger Ratings & 0.10 & {$[-0.09,0.30]$} & $83.9 \%$ & \\
\hline PCL-5 $\leftrightarrow$ Fear Ratings & 0.15 & {$[-0.05,0.34]$} & $92.8 \%$ & \\
\hline PCL-5 $\leftrightarrow$ Hopeless Ratings & 0.22 & {$[0.04,0.41]$} & $98.5 \%$ & $*$ \\
\hline PCL-5 $\leftrightarrow$ Shame Ratings & 0.16 & {$[-0.04,0.35]$} & $93.5 \%$ & \\
\hline PCL-5 $\leftrightarrow$ Powerless Ratings & 0.14 & {$[-0.04,0.34]$} & $92.2 \%$ & \\
\hline PHQ-9 $\leftrightarrow$ Anger Ratings & 0.13 & {$[-0.07,0.33]$} & $89.2 \%$ & \\
\hline PHQ-9 $\leftrightarrow$ Fear Ratings & 0.23 & {$[0.03,0.41]$} & $99.0 \%$ & $* *$ \\
\hline PHQ-9 $\leftrightarrow$ Hopeless Ratings & 0.31 & {$[0.12,0.48]$} & $99.9 \%$ & $* * *$ \\
\hline PHQ-9 $\leftrightarrow$ Shame Ratings & 0.14 & {$[-0.05,0.33]$} & $91.6 \%$ & \\
\hline PHQ-9 $\leftrightarrow$ Powerless Ratings & 0.30 & {$[0.11,0.48]$} & $100.0 \%$ & $* * *$ \\
\hline
\end{tabular}

topics and themes commonly expressed in the essays. The wordcloud in Figure 1a reveals that the most common unigram was 'not,' which suggests that patients tended to write in a negative manner, e.g., about losses, absences, and inabilities. Other common unigrams included 'feel', 'think', and 'people' suggesting themes related to affective, cognitive, and social functioning. The directed graph in Figure 1b elaborates these themes further. Phrases related to safety and trust were particularly common (e.g., 'not feel safe' and 'not trust anyone'), and to a lesser degree so were phrases related to self-esteem (e.g., 'low self esteem'), control/power (e.g., 'no control' and 'too much'), and social comparison (e.g., 'someone else' and 'everyone else'). Patients were explicitly directed to discuss many of these themes by the essay prompt, so the presence of related phrases is not too surprising. However, each theme's representation in the common phrases provides a valuable indication of their relative salience for patients and therefore their relative importance as intervention targets.

The lexical measures generated by our closedvocabulary approach did not correlate very strongly or 


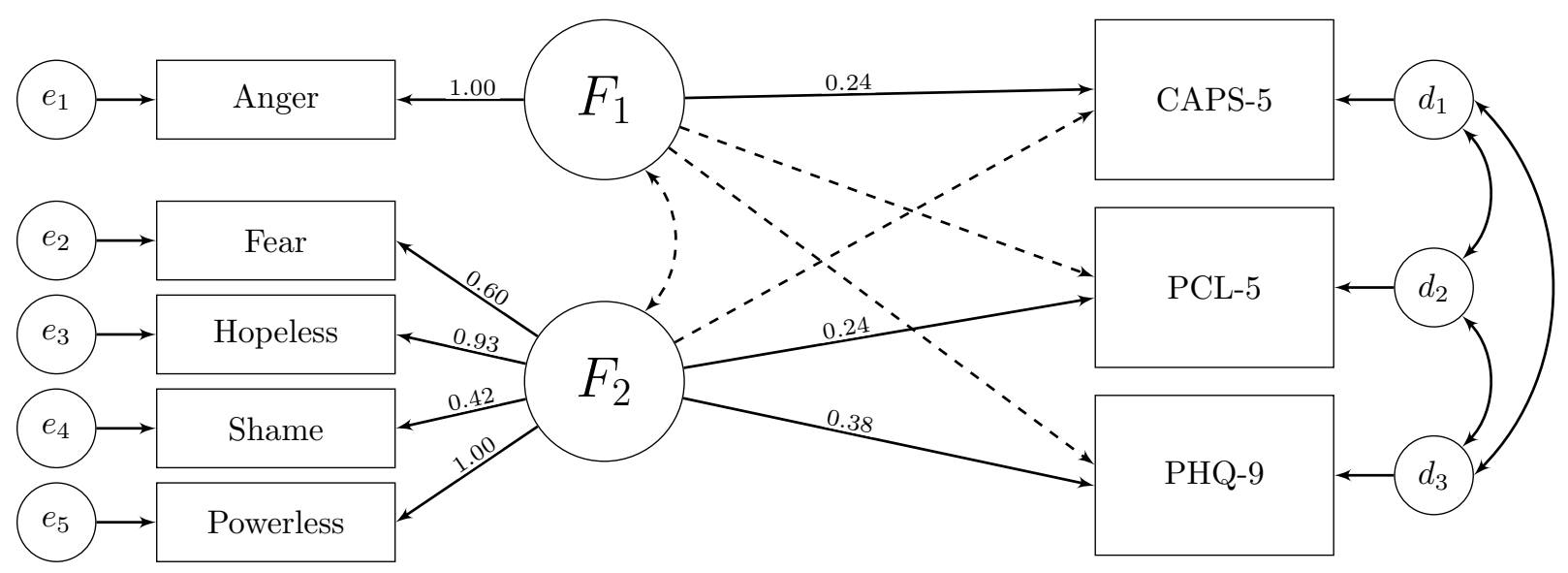

Figure 2

Path diagram for the structural equation model predicting clinical measure total scores from emotion ratings (note that arrows with solid lines are significant or fixed and those with dashed lines are not significant).

consistently with patients' PTSD and depressive symptoms. Out of the ten LIWC categories that we selected on the basis of previous literature and theoretical considerations, only one was significantly associated with a clinical measure. Notably, none of the emotion-related LIWC categories were significantly correlated with patients' symptoms, although this was not the case for the human ratings of expressed emotion ${ }^{4}$. This relatively poor performance may be due to the fact that, as discussed above, the context of negation was essential to properly understanding these essays and LIWC ignores this. Additionally, past studies finding associations between LIWC categories and PTSD symptoms had methodological differences (e.g., focusing on trauma narratives rather than appraisals). The one significant finding in our study using this method was that increased use of plural first-person pronouns was associated with lower self-reported PTSD and depressive symptom severity (but not clinician-rated PTSD symptom severity). This finding may indicate that patients who are more socially engaged (as evidenced by discussing themselves as part of collective groups) tend to be less distressed in general.

The emotional measures generated by our human raters were more strongly and consistently correlated with patients' symptoms. This success was likely driven by the raters' ability to "read between the lines" and consider the text holistically (e.g., in terms of context, intensity, and implicature). Interestingly, the pattern of correlations differed more between clinician-reported and patient-reported symptoms than between PTSD and depressive symptoms. Specifically, expressed anger and shame were positively associated with clinician-rated PTSD symptoms, whereas expressed vulnerability (i.e., fear, powerlessness, and hopelessness) was associated with patientreported PTSD and depressive symptoms. Combining these results with the known limitations of selfreported PTSD symptoms (e.g., Kramer, 2019), we conclude that expressed anger and shame in a patient's trauma appraisal may be particularly useful indicators of their PTSD-specific distress. This interpretation is supported by meta-analytic results showing a particularly strong relationship between PTSD and problematic anger (Olatunji, Ciesielski, \& Tolin, 2010); shame is likely related to negative self-appraisals in PTSD (Leskela, Dieperink, \& Thuras, 2002) and may drive anger in turn (Tangney, Wagner, Fletcher, \& Gramzow, 1992). Expressed vulnerability, on the other hand, may be a more general indicator of nonspecific distress. Future work should explore patients' trauma-related anger in more detail, e.g., by examining who/what they are

\footnotetext{
${ }^{4}$ The correlation between LIWC Negative Emotion words and human-rated negative emotion was small $(\hat{\rho}=0.20)$.
} 
angry at, the appraisals that lead to this anger, angry rumination about the event and its consequences (versus intrusive re-experiencing), how they express anger and attempt to regulate it, and how it may contribute to the maintenance of their PTSD.

Our results join a growing body of research supporting linguistic analysis as a promising technique for clinical research and practice. Asking patients to describe their experiences and symptoms in their own words can be a dynamic and potent complement to traditional questionnaires and rating scales, and linguistic measures can help distill these words for quantitative analysis both within and across individuals. However, our results also highlight the need for careful consideration of the pros and cons of different methods of linguistic analysis. Although convenient and easily scalable, closed-vocabulary approaches like LIWC may lack the sophistication to successfully distill the nuanced information in trauma appraisals and narratives. Human ratings and simple text mining techniques can be superior alternatives for smaller datasets, and more powerful open-vocabulary approaches (e.g., Latent Dirichlet Allocation and Differential Language Analysis; Eichstaedt et al., 2021) hold great promise for larger datasets.

In conclusion, when writing about the causes and consequences of their traumatic experiences, participants often discussed themes of feeling unsafe, having difficulty trusting others, and low self-esteem. Decreased use of plural first-person pronouns and increased expression of vulnerable emotions (e.g., fear, hopelessness, and powerlessness) were associated with higher patient-reported PTSD and depressive symptoms, and increased expression of anger and shame were associated with higher clinician-rated PTSD symptoms. These results underscore the value of interdisciplinary research applying linguistic analysis in clinical psychological science.

\section{References}

Arslan, R. C., Walther, M. P., \& Tata, C. S. (2020). Formr: A study framework allowing for automated feedback generation and complex longitudinal experiencesampling studies using R. Behavior Research Methods, 52, 376-387.

Boudt, K., Cornelissen, J., \& Croux, C. (2012). The Gaussian rank correlation estimator: Robustness properties. Statistics and Computing, 22(2), 471-483.
Brown, T. A. (2015). Confirmatory factor analysis for applied research (Second ed.). Guilford Press.

Ehlers, A., \& Clark, D. M. (2000). A cognitive model of posttraumatic stress disorder. Behaviour Research and Therapy, 38(4), 319-345.

Eichstaedt, J. C., Kern, M. L., Yaden, D. B., Schwartz, H. A., Giorgi, S., Park, G., ... Ungar, L. H. (2021). Closedand open-vocabulary approaches to text analysis: A review, quantitative comparison, and recommendations. Psychological Methods, 26(4), 398-427.

Garnier-Villarreal, M., \& Jorgensen, T. D. (2020). Adapting fit indices for Bayesian structural equation modeling: Comparison to maximum likelihood. Psychological Methods, 25(1), 46-70.

Gelman, A., Carlin, J. B., Stern, H. S., Dunson, D. B., Vehtari, A., \& Rubin, D. B. (2014). Bayesian data analysis (3rd ed.). CRC Press.

Iverson, K. M., King, M. W., Cunningham, K. C., \& Resick, P. A. (2015). Rape survivors' trauma-related beliefs before and after Cognitive processing therapy: Associations with PTSD and depression symptoms. Behaviour Research and Therapy, 66, 49-55.

Jaeger, J., Lindblom, K. M., Parker-Guilbert, K., \& Zoellner, L. A. (2014). Trauma narratives: It's what you say, not how you say it. Psychological Trauma: Theory, Research, Practice, and Policy, 6(5), 473.

Kleim, B., Horn, A. B., Kraehenmann, R., Mehl, M. R., \& Ehlers, A. (2018). Early linguistic markers of traumaspecific processing predict post-trauma adjustment. Frontiers in Psychiatry, 9, 645.

Kramer, L. (2019). Self-rated versus clinician-rated assessment of posttraumatic stress disorder: An evaluation of diagnostic discrepancies between the PTSD Checklist for DSM-5 (PCL-5) and the ClinicianAdministered PTSD Scale for DSM-5 (CAPS-5) (Unpublished doctoral dissertation). Auburn University, Auburn, AL.

Kroenke, K., Spitzer, R. L., \& Williams, J. B. W. (2001). The PHQ-9. Journal of General Internal Medicine, 16(9), 606-613.

Leskela, J., Dieperink, M., \& Thuras, P. (2002). Shame and posttraumatic stress disorder. Journal of Traumatic Stress, 15(3), 223-226.

Makowski, D., Ben-Shachar, M. S., Patil, I., \& Lüdecke, D. (2020). Methods and algorithms for correlation analysis in R. Journal of Open Source Software, 5(51), 2306.

McGraw, K. O., \& Wong, S. P. (1996). Forming inferences about some intraclass correlation coefficients. Psychological Methods, 1(1), 30-46.

McKinney, J. M., Hirsch, J. K., \& Britton, P. C. (2017). PTSD symptoms and suicide risk in veterans: Serial indirect effects via depression and anger. Journal of 
Affective Disorders, 214, 100-107.

Merkle, E. C., \& Rosseel, Y. (2018). Blavaan: Bayesian structural equation models via parameter expansion. Journal of Statistical Software, 85(1), 1-30.

Miragoli, S., Camisasca, E., \& Di Blasio, P. (2019). Investigating linguistic coherence relations in child sexual abuse: A comparison of PTSD and non-PTSD children. Heliyon, 5(2), e01163.

Olatunji, B. O., Ciesielski, B. G., \& Tolin, D. F. (2010). Fear and loathing: A meta-analytic review of the specificity of anger in PTSD. Behavior Therapy, 41(1), 93-105.

Papini, S., Yoon, P., Rubin, M., Lopez-Castro, T., \& Hien, D. A. (2015). Linguistic characteristics in a non-trauma-related narrative task are associated with PTSD diagnosis and symptom severity. Psychological Trauma: Theory, Research, Practice, and Policy, 7(3), 295-302.

Park, C. L. (2010). Making sense of the meaning literature: An integrative review of meaning making and its effects on adjustment to stressful life events. Psychological Bulletin, 136(2), 257-301.

Pennebaker, J. W., Boyd, R. L., Jordan, K., \& Blackburn, K. (2015). The development and psychometric properties of LIWC2015. University of Texas at Austin.

Pennebaker, J. W., Mehl, M. R., \& Niederhoffer, K. G. (2003). Psychological aspects of natural language use: Our words, our selves. Annual Review of Psychology, 54, 547-77.

Resick, P. A., Monson, C. M., \& Chard, K. M. (2017). Cog- nitive processing therapy for PTSD: A comprehensive manual. Guilford Press.

Schein, J., Houle, C., Urganus, A., Cloutier, M., PattersonLomba, O., Wang, Y., ... Davis, L. L. (2021). Prevalence of post-traumatic stress disorder in the United States: A systematic literature review. Current Medical Research and Opinion, 37(12), 2151-2161.

Silge, J., \& Robinson, D. (2021). Text mining with R: A tidy approach. O'Reilly.

Sobel, A. A., Resick, P. A., \& Rabalais, A. E. (2009). The effect of cognitive processing therapy on cognitions: Impact statement coding. Journal of Traumatic Stress, 22(3), 205-211.

Tangney, J. P., Wagner, P., Fletcher, C., \& Gramzow, R. (1992). Shamed into anger? The relation of shame and guilt to anger and self-reported aggression. Journal of Personality and Social Psychology, 62(4), 669675.

van der Loo, M. P. (2014). The stringdist package for approximate string matching. The R Journal, 6(1), 111.

Weathers, F. W., Blake, D. D., Schnurr, P. P., Kaloupek, D. G., Marx, B. P., \& Keane, T. M. (2013). The Clinician-Administered PTSD Scale for DSM-5 (CAPS-5). National Center for PTSD.

Weathers, F. W., Litz, B. T., Keane, T. M., Palmieri, P. A., Marx, B. P., \& Schnurr, P. P. (2013). The PTSD Checklist for DSM-5 (PCL-5). National Center for PTSD. 